\title{
Material Analyses of Foam-based SiC FCI after Dynamic Testing in PbLi in MaPLE Loop at UCLA
}

\author{
María González ${ }^{\mathrm{a} *}$, David Rapisarda ${ }^{\mathrm{a}}$, Angel Ibarra ${ }^{\mathrm{a}}$, Cyril Courtessole $^{\mathrm{b}}$, Sergey Smolentsev $^{\mathrm{b}}$, \\ Mohamed Abdou ${ }^{\mathrm{b}}$
}

LNF-CIEMAT, Avda Complutense, 40. 28040 Madrid, Spain
${ }^{b}$ Fusion Science and Technology Center, UCLA, USA

\begin{abstract}
Foam-based SiC flow channel inserts (FCIs) developed and manufactured by Ultramet, USA are currently under testing in the flowing hot lead-lithium (PbLi) alloy in the MaPLE loop at UCLA to address chemical/physical compatibility and to access the MHD pressure drop reduction. UCLA has finished the first experimental series, where a single uninterrupted long-term ( $\sim 6500$ hours) test was performed on a 30 -cm FCI segment in a magnetic field up to $1.8 \mathrm{~T}$ at the temperature of $300^{\circ} \mathrm{C}$ and maximum flow velocities of $\sim 15 \mathrm{~cm} / \mathrm{s}$. After finishing the experiments, the FCI sample was extracted from the host stainless steel duct and cut into slices. Few of them have been analyzed at CIEMAT as a part of the joint collaborative effort on the development of the DCLL blanket concept in the EU and the US. The initial inspection of the slices using optical microscopic analysis at UCLA showed significant PbLi ingress into the bulk FCI material that resulted in degradation of insulating properties of the FCI. Current material analyses at CIEMAT are based on advanced techniques, including characterization of FCI samples by FESEM to study PbLi ingress, imaging of cross sections, composition analysis by EDX and crack inspection. These analyses suggest that the ingress was caused by local defects in the protective inner CVD layer that might be originally present in the FCI or occurred during testing.
\end{abstract}

Keywords: FCI Development, Dual Coolant Lead Lithium breeder blanket concept, DCLL, Silicon Carbide, SiC, Material Characterization

\section{Introduction}

The Dual Coolant Lead Lithium (DCLL) breeding blankets have been proposed for a DEMO reactor, where liquid lead-lithium alloy (PbLi) is used for power conversion and tritium breeding. A SiC-based Flow Channel Insert (FCI) is used to reduce the magnetohydrodynamic (MHD) pressure drop from the flowing liquid and as a thermal insulator to separate the high-temperature $\mathrm{PbLi}\left(700^{\circ} \mathrm{C}\right.$ and higher) from the helium-cooled RAF/M steel structure [1].

The MHD flow facility MaPLE (Magntohydrodynamic PbLi Experiment) at UCLA, that utilizes molten $\mathrm{PbLi}$ alloy as working fluid, is a unique US facility that allows for testing the performance of FCIs under a magnetic field at working conditions relevant to the DCLL breeding blanket.

$\mathrm{SiC}$ is considered as a good candidate material for high temperature FCI applications. It is expected to provide both the required electrical and thermal insulation between high-temperature liquid $\mathrm{PbLi}$ and the structural RAF/M material. Electrical insulation mitigates the MHD pressure drop, which would otherwise result in intolerably high stresses in the structural walls.

The long-term objective of the collaboration between UCLA and CIEMAT is to support the development of designs and technologies linked to DCLL concept. The objective of the experiments performed at UCLA was to characterize the performance of foam-based CVD-coated $\mathrm{SiC}$ material in the conditions of the flowing $\mathrm{PbLi}$ in a magnetic field. The goal of the accompanying studies at CIEMAT, which are reported in this paper, was to conduct material analysis of the FCI segment used in the experiments.

\section{Dynamic testing of FCI at UCLA and material characterization}

\subsection{Dynamic testing of FCI in MaPLE}

The MHD PbLi flow loop at University of California, Los Angeles (UCLA) called MaPLE was constructed with the main objective of addressing PbLi behavior in blanket relevant conditions to take into account the effect of a magnetic field [2]. The loop operation parameters are: maximum magnetic field $1.8 \mathrm{~T}, \mathrm{PbLi}$ temperature up to $350^{\circ} \mathrm{C}$, maximum $\mathrm{PbLi}$ flow rate with/without a magnetic field $15 / 50 \mathrm{l} / \mathrm{min}$, maximum pressure head 0.15 $\mathrm{MPa}$.

An MHD pressure drop in recent experiments on testing a foam-based SiC FCI was measured in PbLi flows in a host stainless steel rectangular duct where the FCI was inserted. The FCI fabricated by Ultramet, Inc. has a square shape with rounded corners. It is sealed on both inner and outer surfaces with dense $\mathrm{SiC}$ facesheets to protect from PbLi ingress (Fig. 1). As an additional protection means, the bulk $85 \%$ porosity material is filled with silica aerogel. The pressure drop was

maria.gonzalez@ciemat.es 
measured over the bare duct section (without the FCI) and over the $30 \mathrm{~cm}$ FCI segment simultaneously, using an indirect pressure measurement technique described in [2]. The magnetic field in the experiment was varied from zero to $1.8 \mathrm{~T}$ (Hartmann number up to 1500) and the velocity up to $\sim 15 \mathrm{~cm} / \mathrm{s}$ (Reynolds numbers above 5000). The uninterrupted test of the FCI took approximately 6500 hours. Further details describing the loop and the experiments could be found elsewhere [3]. Surprisingly, the experiments at UCLA did not demonstrate anticipated reduction of the MHD pressure drop, which could be explained by $\mathrm{PbLi}$ ingress causing degradation of FCI electrical insulation. As a matter of fact, weight analysis, visual inspection and preliminary microscopic analysis at UCLA all confirmed significant $\mathrm{PbLi}$ ingress equivalent to about $45 \%$ of the total $\mathrm{FCI}$ volume.

(a)

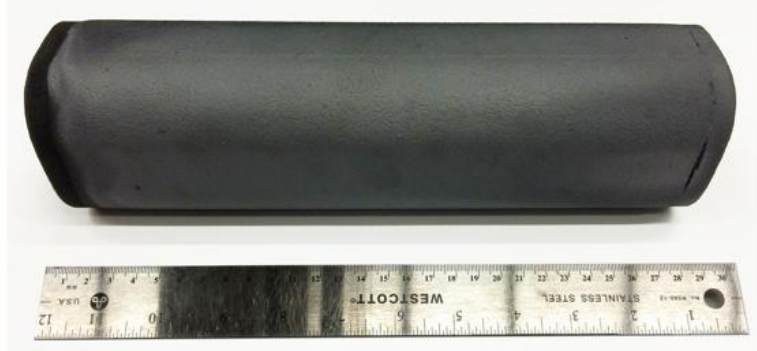

(b)

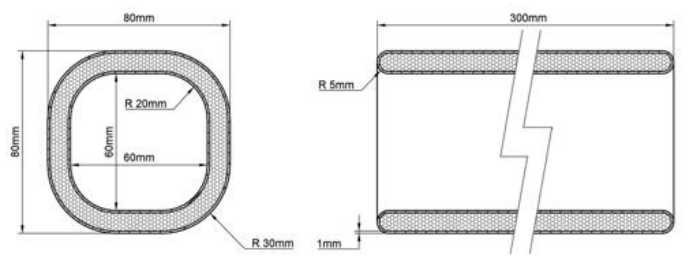

Fig. 1. Foam-based SiC FCI with CVD coating manufactured by Ultramet, USA: (a) a 30-cm FCI segment and (b) overall FCI segment dimensions.

\subsection{Post-testing material characterization}

Several samples were produced from the FCI segment used in the experiments at UCLA with a diamond saw, from the inlet, middle and outlet sections of the FCI box. These samples were analyzed at CIEMAT for cracking and other possible defects, internal microstructure and elemental composition to figure out possible causes of the ingress. As a first approach, a Nikon optical microscope was used. After further cutting and mirror-like diamond polishing, samples were C-coated to avoid surface charging due to the electron beam. A Zeiss Auriga Compact scanning electron microscope (FESEM+FIB) and a Bruker XFlash EDX detector were applied for imaging and chemical analysis. All FESEM images presented here in Figs. 2, 3 and 5 show the background in black, the $\mathrm{SiC}$ and the filler phases in grey and the solidified PbLi in bright white. Few samples were also FESEM studied asfractured from the as-received condition.

\section{Results and Discussion}

Heavy $\mathrm{PbLi}$ ingress was observed to occur during testing in the flowing liquid in a fresh fractured FESEM image (Fig. 2, top). Elemental lead was detected both filling the pores and also the core of the SiC ligament structure itself, as seen in the magnified SEM image (Fig. 2, bottom). The EDX mappings or line profile analyses show the purity of $\mathrm{SiC}$ fibers, the presence of Si-based granules inside the pores of the 3D foam structure, together with the ingress of PbLi (Fig. 3).
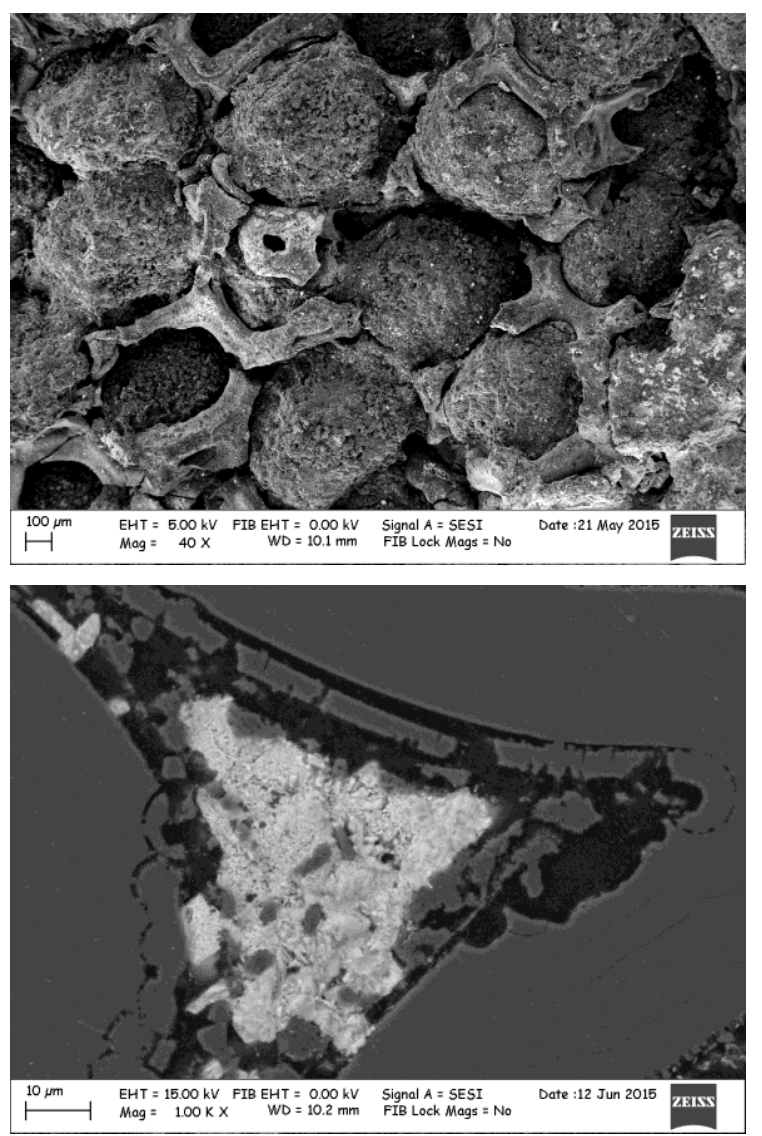

Fig 2. Fresh fracture FESEM image taken from the inlet section of the SiC FCI tube showing the FCI foam filled (top). $\mathrm{PbLi}$ was also detected filling the core of the $\mathrm{SiC}$ ligaments (bottom).

The samples taken from the FCI inlet section demonstrate more ingress compared to those samples taken from the middle and outlet sections, which show about the same ingress. A measurement of the center of mass location showed that it coincided with the geometrical center of the FCI. This suggests a uniform ingress over the entire FCI length. The post-testing examination also indicates an occasional lack of the facesheet material mainly in the inlet sample, which could be due to manufacturing flaws or might occur in the course of testing since the experiment took unexpectedly an unusual long time (about 6500 hours). However, since testing was not interrupted for examination of the FCI, the reason for defects in the 
CVD layer is not completely understood at this moment. Furthermore, the inner facesheet was found to be thinner and at some locations was completely absent, while the outer CVD layer almost did not change its nominal thickness of about $1 \mathrm{~mm}$. The defects in the inner CVD coating should be then considered as the most possible way of $\mathrm{PbLi}$ ingress into the foam structure. Since previous immersion tests in static molten $\mathrm{PbLi}$ on uncoated $\mathrm{SiC}$ samples also manufactured by Ultramet exhibited minimal metal ingress at $700^{\circ} \mathrm{C}$ [3], the explanation for the observed ingress in dynamic tests could be related to the flow effects. Moreover, in the dynamic tests the FCI sample was exposed to PbLi over a very long period of about 6500 hours while static tests took only 100 hours.
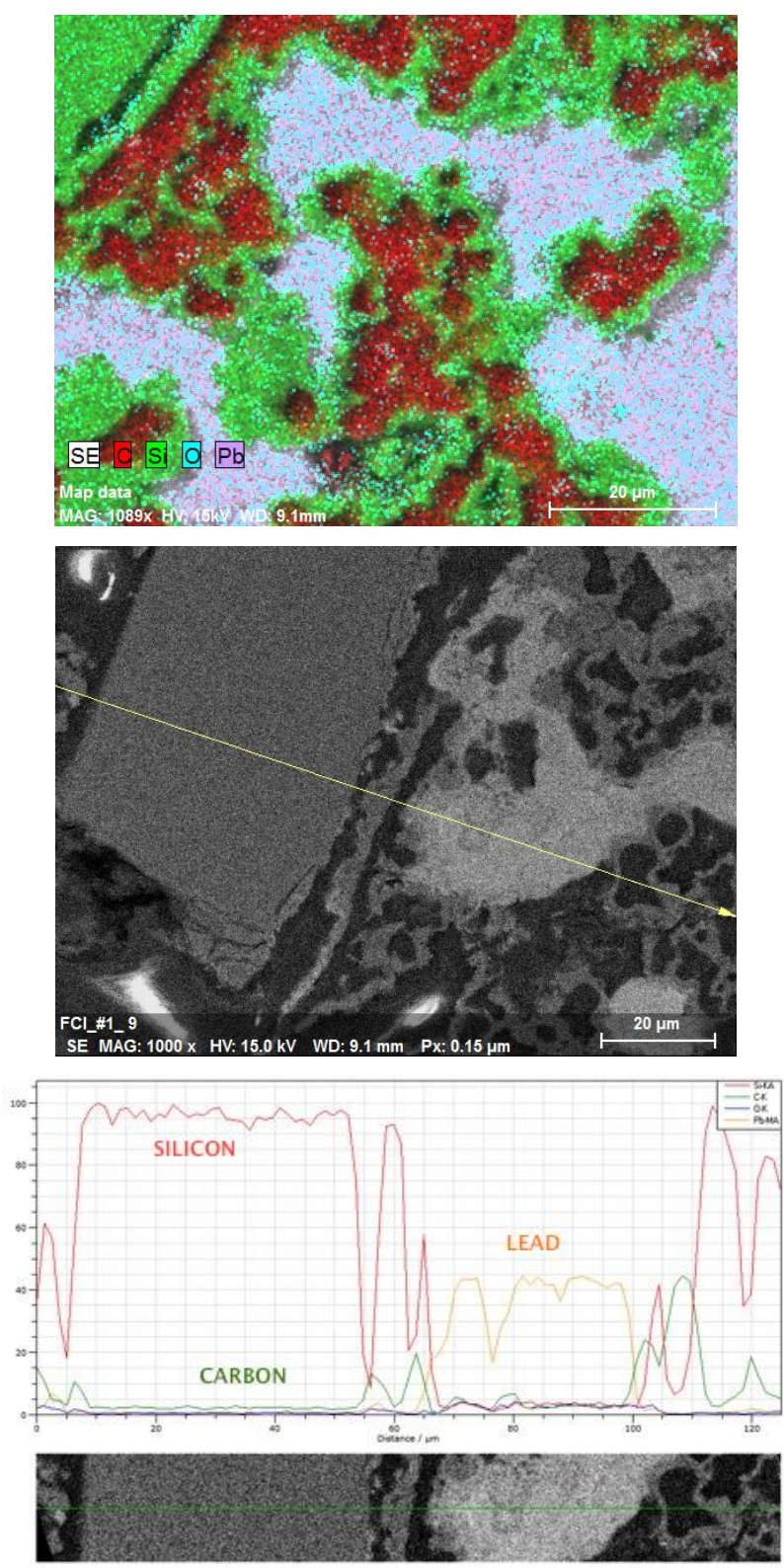

Fig 3. EDX mapping showing the elemental composition of a FCI cross-section sample (top figure). The analytical line profile (bottom figure) crosses the $\mathrm{SiC}$ foam structure into the infiltrated pores also filled with solidified $\mathrm{PbLi}$.
Highly cracked samples were found when studying the microstructure; the cracks have been observed to run through both the $\mathrm{SiC}$ dense coatings and also through the foam $\mathrm{SiC}$ structure. Cracking in the FCI appeared visually in a couple of weeks after it was extracted from the liquid metal loop, possibly due to the exposure of the FCI to the air and atmospheric moisture. A few larger cracks also formed when the FCI segment was being sawed into slices. All cracks, large and microscopic, were not filled with $\mathrm{PbLi}$ suggesting that no crack formation occurred during the experiment.

No difference in the PbLi ingress between the Hartmann and the side walls (nomenclature given to the FCI walls located perpendicular and parallel to the magnetic field, respectively) was found as seen from the micrographs. This clearly demonstrates that there is no effect of the magnetic field direction on the PbLi transport into the FCI bulk material and also on corrosion/erosion of the protective CVD layer. Nevertheless, the solidified metallic phase seems to be more concentrated in regions next to the CVD coating. In particular, more ingress was found near the outer FCI surface compared to the inner surface. Figure 4 shows the plot of the PbLi-to-SiC phase balance obtained with the ImageJ free software applied to a number of average images taken from the outlet section. The reason for higher $\mathrm{PbLi}$ concentration near the CVD layers is not clear but it could be related to the radial temperature gradient during the FCI segment cooling after extracting the FCI from the host duct. The rather low volume of solidified PbLi may generate a pressure gradient, which could favor the liquid solidification near the dense SiC.

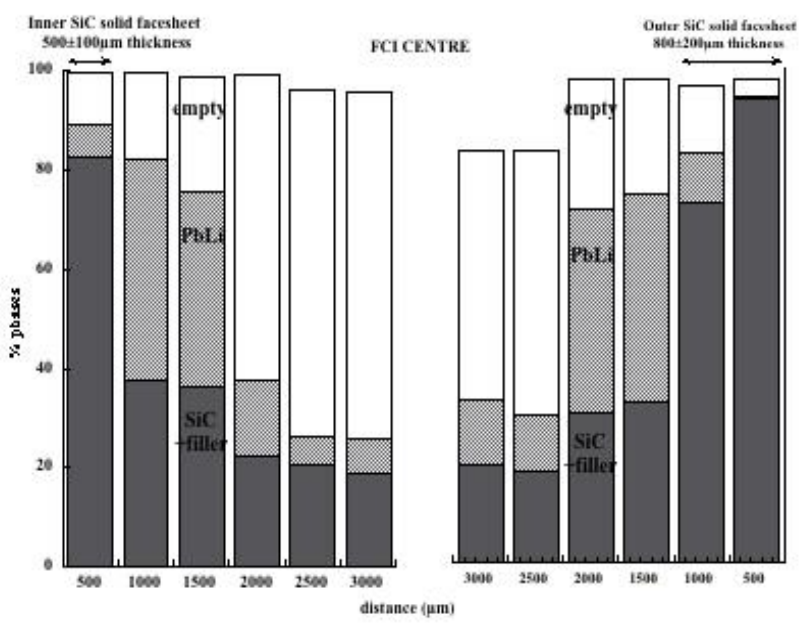

Fig 4. Local distribution of the solid phases across the FCI wall (from the inner to the outer facesheet) after the MHD experiment in PbLi. "Empty" refers to the remaining empty space in the foam pores. The measurement errors mostly appear when calculating the empty space.

Magnified images suggest that no damage occur to both the surface of the $\mathrm{SiC}$ facesheets and the foam constituents in the flowing $\mathrm{PbLi}$ run (Fig. 5). Furthermore, the EDX line profiles indicate no lead 
diffusion into the SiC phase and the absence of oxygen, nitrogen, as common impurities that could be introduced during the experiment. This suggests good chemical compatibility between $\mathrm{PbLi}$ and dense $\mathrm{SiC}$, at least in the experimental conditions. Therefore, the observed thinning of the inner CVD layer is likely related to the manufacturing process rather than corrosion/erosion. Solidified $\mathrm{PbLi}$ and metal oxide particles showing different geometries (rounded drops, needle- or plateletlike particles, see also figure 5) have been found adhered rarely to the surfaces of the foam ligaments and to the dense $\mathrm{SiC}$ facesheets. Oxidation of $\mathrm{PbLi}$ probably occurred along with the development of cracks.
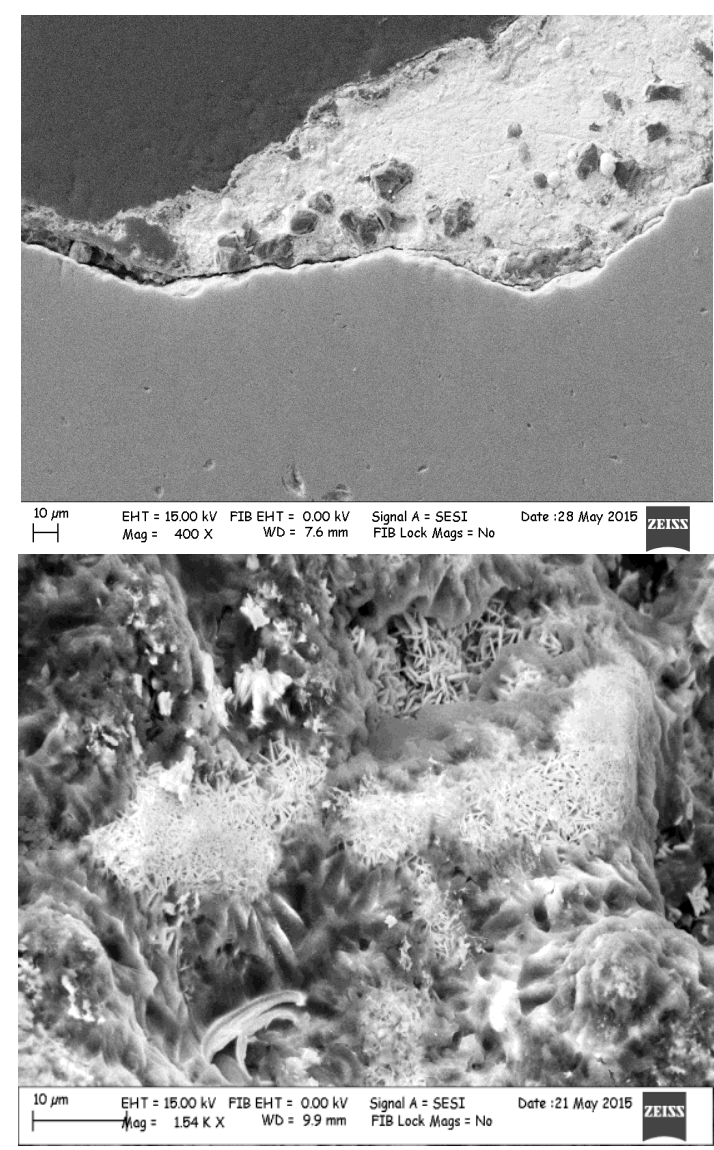

Fig 5. Top) PbLi or lead oxide particle adhered to the inner FCI coating showing no SiC surface modification. Left) needle-like $\mathrm{PbLi}$ particle decorating the surface of an internal $\mathrm{SiC}$ foam ligament.

\section{Conclusions}

After finishing dynamic experiments in the flowing hot $\mathrm{PbLi}$, samples from the inlet, middle and outlet sections of the SiC-coated, foam-based $\mathrm{SiC}$ FCI infiltrated with silica aerogel were analyzed by looking at their SEM microstructure and elemental composition.

These analyses confirmed heavy PbLi ingress that could be explained by liquid metal infiltration through local defects in the protective CVD layer. Most likely most of the infiltration occurred through the inner CVD facesheet, which was found to be much thinner than the outer one. Since no direct evidences of corrosion/erosion were observed, these defects could be related to the manufacturing process. If so, next efforts on development and fabrication of coated SiC FCIs should focus more on protective layers that guarantee no liquid metal ingress over a long run.

\section{Acknowledgments}

This study was performed as a part of the collaborative agreement between UCLA and CIEMAT on the development of the DCLL blanket concept in the EU and the US. The material analyses reported in this paper have been carried out within the framework of the EUROfusion Consortium and have received funding from the Euratom research and training programm 20142018 under grant agreement No 633053. The views and opinions expressed herein do not necessarily reflect those of the European Commission. The microstructural study was performed using instrumentation partially funded with the European Regional Development Fund (FEDER-ICTS-2011-06). The US authors acknowledge support from the US Department of Energy, Office of Fusion Energy Sciences, under Grant No. DE-FG0286ER52123.

\section{References}

[1] S. Malang, M. Tillack, C. P. C. Wong, N. Morley, S. Smolentsev, Development of the lead lithium (DCLL) blanket concept, Fusion Sci. Technol. 60 (2011) 249-256.

[2] S. Smolentsev, F-C. Li, N. Morley, Y. Ueki, M. Abdou, T. Sketchley, Construction and initial operation of MHD $\mathrm{PbLi}$ facility at UCLA, Fusion Engineering and Design 88 (2013) 317-326.

[3] S. Smolentsev, C. Courtessole, M. Abdou, S. Sharafat, S. Sahu, T. Sketchley, First results of static and dynamic testing of foam-based $\mathrm{SiC}$ flow channel insert in leadlithium. Submitted to J. Nuclear Materials, 2015. 